LENNARTSSON, C. y SILVERSTEIN, M. (2001). Does engagement with the life enhance survival of elderl people in Sweden? Journal of Gerontology, 56b(6) 335-342

MCDONALD, J. (1996). Community participation in an Australia retirement village. Australian Journal on Ageing, 25(1), 167-171

MCGUINN, K. y MOSHER, A. (2000). Participation in recreational activities and its effect on perception of life atisfaction on residential settings. ion, and Aging, 25(1), 77-86

ROWE, J. W. y KAHN, R. L. (1997). Successful aging. The Gerontologist, 37, 433-440.

SEEMAN, T. (2000). Health promoting effects of friends comes in older adult. American Journal Health Promotion, 14(6), 362-370.

SENAMA. (2003). Chile necesita a sus Adultos Mayores: Participación social. Santiago de Chile: Servicio Nacional del Adulto Mayor

TRIADÓ, C., CELDRÁN, M., CONDE, L. y OTROS (2008). Envejecimiento productivo: la provisión de cuidados de los abuelos a los nietos. Implicaciones

VEGA, J., BUZ, J. y BUENO, B. (2002). Niveles de actividad y participación social en las personas mayores de 60. Revista Interuniversitaria de Formación de Profesorado, 45 .

WARBURTON, J., FERRY, D. J., ROSENMAN, L. S. y OTROS (2001). Differences between older volunteers and nonvolunteers. Research on Aging, 23(5), 586605.

WARBURTON, J. y STIRLING, C. (2007). Factors affecting volunteering among older rural and city dwelling adults in Australia. Educational Gerontology, 33(1), 23-43.

\title{
Gestión del deseo en la intervención social: claves estéticas y el riesgo de la participación
}

The management of desire in Social practice: Aesthetic key and the Risk of participation

JaVIERA ROA INFANTE

Magister en Trabajo Social, PUC. Marín No30, Santiago Centro. correo electrónico: jiroa@uc.cl.

\section{Resumen}

Existe un creciente interés desde las politicas sociales por incorporar al otro (usuario, beneficiario poblador, etc.) en la intervención social: su opinión, inquietudes, intereses. Muchas veces, esta intención aparece bajo la categoria de participación, la que intenta ser instalada como modo de hacer en procesos desplegados por el Estado, por privados y su RSE, por gobiernos locales y ONGs Este documento muestra cómo estas buenas intenciones participativas se vuelven peligrosas, si es que no asumen el desafio de incorporar al otro en su complejidad, generándose muchas veces respuestas anestesiadas, que no hacen más que reducir o anular su condición de sujeto. De esta formestas anestesiadas, que no hacen más que reducir o anular su condición de sujeto. De esta desde la critica a la participación se propone como ejemplo para abrir la categoria del deseo nerando nuevas posibilidades de trabajo, a través de lo que hemos denominado la "gestión de deseo en la intervención social.

Palabras clave. Participación, estética, intervención social, sujeto, deseo.

\section{Abstract}

There is a growing interest from social policy to incorporate the other (user, beneficiary, resident, etc.) in social practice: its view, concerns, interests. Many times, this intention appears under the category of participation, which is intended to be installed as a way of doing in processes undertaken by the State, by private and their CSR, local governments and NGOs.

This document will show how these participatory good intentions become dangerous, if it is not assumed the challenge of incorporating the other in their complexity, often generating responses anesthetized, which only serve to reduce or denie their status as subjects.

Thus, the criticism to participation is proposed as an example to open a category of desire from the aesthetic dimension of social practice -negative rational display- generating new employ-

ment opportunities, through what we will call the "management desire for social practice".

Key words. participation aesthrtic, social practice, subject, desire.

Los riesgos de la participación

Cada vez más se exige a quienes implementa diseña políticas sociales y procesos de intervención social incorporar a los afectados en la solución de sus problemas. Muchas veces este asunto se resuelve con el requerimiento de hacer participar a la gente. Presupuestos participativos, consultas ciudadanas, mesas de trabajo, son algunas de las metodologías que se despliegan en estos términos, dibujándose una linea de tendencia de las policies, fortalecida por un amplio consenso a nivel nacional respecto de la importancia de la participación social de cara al fortalecimiento de la ciudadania, la democracia el ejercicio de las políticas públicas (Serrano, 1998:1)

¿Por qué esto puede ser riesgoso? Si más allá de la retórica participativa algún modelo de intervención logra hacer efectivamente parte al usuario en el proceso, ¿cómo podría ser en alguna medida problemático? Y es que incorporar al otro en la intervención social enfrenta el desafío de la complejidad del otro. No es posible su resolución con mecanismos lineales y unidimensionales, porque 
ducción de su lugar en el mundo. No podemos incorporarlo participativamente al precio de su condición de sujeto.

\section{Cumplir lo que la gente quiere o el \\ sujeto por la borda}

Se darán cuatro ejemplos recogidos de la experiencia como trabajadora social de la autora, donde es posible problematizar esto que se ha denominado "los riesgos de la participación".

1. "Con esto de la participación la gente se ha puesto regodiona y pedigüeña" decía una profesional que desempeña el cargo de trabajadora social en un municipio. Y es que con una incorporación del otro reducida, es posible terminar cargados con listas de petitorios, lo que se perfila -sobre todo en contextos municipales en años de elección- en una amenaza al acecho para los profesionales y los procesos de intervención. Por otra parte, se desencadena un proceso de frustración del usuario cuando no tiene lo que pide: nada peor que un cliente insatisfecho. 2. Trabajando temáticas de embarazo adolescente con jóvenes mujeres de entre 13 y 18 años infractoras de ley con consumo problemático de drogas, fue posible percatarse de que la mayoría de a eran madres, y todas así lo habian querido. Para ninguna el embarazo había sido un accidente, sino algo que ellas deseaban. Nuestros dispositivos de intervención trabajaban desde la lógica del embarazo no deseado con niñas y adolescentes que deseaban ser madres, porque en el contexto social que ellas se mueven la maternidad es -entre otras cosas- un hito de validación de la condición de mujeres. Su aspiedad y les permitiera salir de sus casas donde sufran las principales vulneraciones a sus derechos; siendo en casos su deseo ser madre de algún joven de la población que fuera altamente valorado dentro de a cultura delictual: el choro más choro. Ellas sabían perfectamente cómo usar un sistema anticonceptivo. ¿Cómo conseguir que los procesos de transformación en las posibilidades de estas niñas no provengan de la imposición externa, sino que sean ellas las que deseen un horizonte diferente? ¿Cómo accionar nuevas posibilidades sin que el precio sea su condición de sujeto?

3. Incluso en los dispositivos más sofisticados de participación, como es el caso de las Mesas de

Trabajo $^{1}$ de la Fundación Proyecto Propio, esta problemática se expresa. Mediante una muy participativa metodología, esta Fundación propone a a comunidad en los sectores de más alta den s alta densidad habitacional en conjuntos de vivienda social de la Región Metropolitana ${ }^{2}$ desarrollar un proyecto que la misma comunidad quiera hacer (de ahí el nombre Proyecto Propio): ¿qué desea hacer la gente en estos contextos para mejorar sus vidas?

Un lugar común -la mayoría de las veces proveniente de un grupo de esforzadas mujeres vecinas del sector donde la autora se encontraba trabajando- fue la respuesta: "... queremos hacer (o mejorar) do- fue la respuesta: "... queremos hacer (o mejorar)
una "cancha" para "sacar a los jóvenes de la droga"..." El proceso de trabajo dura aproximadamente 8 semanas, donde los vecinos formulan y ejecutan el proyecto, aportando entre un 30 y 50 porciento de su costo. Tiempo después, era posible visualizar al grupo de jóvenes de movilizado a estas comprometidas mujeres- consumiendo drogas en la cancha nueva, que ellas habían implementado, levantando fondos con completadas y rifas. De ahí vendría la rabia, la desconfianza y el desencanto: "si con estos cabros no se puede, nos esforzamos por ellos y mira, ahí siguen consumiendo droga en la cancha nueva" y con ello la pérdida del interés por trabajar en conjunto en mejoras para la vida en comunidad. El problema era grave.

Otro proyecto recurrente era construir un cierre perimetral o reja en el block para aumentar la seguridad. Aquí las paradojas son alarmantes: en uno de los barrios, la noche anterior a la entrega de los departamentos de una villa nueva, los vecinos de la parte de los departamentos nuevos, porque no querían que "delincuentes" llegaran a vivir a su sector. Por su parte, los nuevos residentes del barrio, al enterarse de lo sucedido, temiendo por su seguridad aceptaron ir a vivir a la nueva villa porque era eso, o nada. Sumado a esto, los datos disponibles indican que la violencia en estos barrios proviene principalmente del fenómeno de violencia doméstica, donde como proceso de intervención se agrega una fractura más a la ya fracturada ciudad y sus relaciones de colaboración

Ahora bien, ¿Por qué no construir la reja, si eso es lo que la gente quiere y lo que el proceso participativo ha arrojado como resultado?

Metodología de trabajo comunitario desarrollada por Fundación Proyecto Propio. Mediante reuniones semanales y el acompañamiento de tableros de papel, se desarrolla un proceso de autodiagnóstico, planificación y evaluación de un proyecto que la comunidad decida desarrollar en post de su mejora

2 Bajos de Mena en Puente Alto, Sol Poniente en Maipú, San Luis en Quilicura.
Estas paradojas buscan ejemplificar la insuficiencia en la incorporación del otro y sus deseos en la intervención social. ¿Debe una política participativa responder a lo que sea que la gente quiere? ¿Quiénes son los profesionales para discernir que esto que se quiere sí, romper estas relaciones mediatizadas que se instalan en lo que la gente quiere? ¿Cómo abrir posibilidades de que amplien su propio espectro de lo que es posible/imposible?

Aparece la importancia de "gestionar el deseo"; propuesta que se desarrolla a continuación, frente a la evidencia de incomodidades insalvables cuando se respuestas contundentes desde la intervención social a la incorporación del otro y su despliegue como sujeto.

\section{Gestionar el deseo ${ }^{3}$, explorando desde} la dimension estetica la incorporacion del otro en el proceso

Hablar del deseo en la intervención social desde la apertura de su dimensión estética es un asunto complejo y complicado. Se partirá despejando las complicaciones con algunas aclaraciones iniciales -que no resuelven el tema, pero instalan un piso mínimo para abrir la categoría-, respecto de la noción de estética y luego de la categoría de deseo, para -posteriormente- hacerse cargo de aquello que interesa: su complejidad.

\section{Cuatro mitos sobre la noción de estética}

Adentrarse en la dimensión estética de la intervención social implica abrirse al concepto más allá de lo que el sentido común ha instalado, y romper algunas prenociones existentes. A continuación 4 mitos sobre la noción de estética:

a. El mito de la homologación arte- estética Quizá, para muchos de los lectores arte y estética son conceptos que no es posible distinguir. Y es que se le ha dado una importancia exagerada a la producción artística en el discurso dominante de la estética (Mandoki, 2006: 20), volviéndose arte y estética en sinónimos, un mito de fuerte arraigo. La estética académica ha considerado y especificado el estatus privilegiado de ciertos objetos - las obras de artehistoriadores del arte- como ámbitos atingentes a la estética aunque existan muchos otros objetos, sujetos y sucesos pertinentes a esta disciplina. Desde la perspectiva de Mandoki, el estetólogo insiste en

\footnotetext{
Estas reflexiones se insuman de la tesis que la autora desarrolló para optar al grado de magister en Trabajo Social en la Pontifici
Universidad Católica de Chile: "La dimensión estética de la intervención social, aportes en un contexto de barbarie en la cultura"
}

seguir trabajando en los museos, bibliotecas y sala de arte con sus libros, partituras y cuadros, para no ser turbado por los olores, sudores y ardores de la vida cotidiana (Mandoki, 2006: p. 25).

"El mito de que el arte sea exclusivamente estético ha servido para reproducir la idea de la producción artística como desvinculada de todo interés o utilidad social fuera de la función contemplativa, y a artista como un genio dotado de una sensibilidad prodigiosa. (...) el arte no es exclusivamente estético porque esta dimensión de ninguna manera agota la variedad de formas de relación con el arte. Existen también formas de relación técnica en la verificación de autenticidad de una obra, epistemológicas, políticas, publicitarias, psicológicas, terapéuticas, financieras..." (Mandoki, 2006: 43).

Con esta afirmación, la autora abre la posibilidad del encuentro de lo estético con otros objetos y sujetos de conocimiento, abriendo la posibilidad de encuentro estética- intervención social.

b. El mito de la reducción de la estética a lo bello Esta reducción que homologa estética con lo bello se relaciona -por una parte- con el mito de la homologación arte-estética recién expuesto, donde entra en juego una segunda reducción que es que el objeto del arte es lo bello. Christopher S. Nwodo argumenta en este sentido, indicando que la estética vendría a ser la teoría que estudia la belleza, relegando el arte a la teoría del arte. El problema con la propuesta de Nwodo no es tanto que lo bello haya perdido en el arte contemporáneo el papel dominante que tenía en el arte académico; el problema es que, como categoría, lo bello por si mismo no tiene relevancia suficiente para fundar una disciplina Mandoki, 2006)

Para Adorno, "hacer de la estética una doctrina sobre la belleza es infecundo porque el concepto de belleza nace del conjunto del contenido estético" (Adorno, 1971: 73). Lo bello no puede definirse, pero tampoco se puede renunciar a su concepto, pues más es algo que se capta, que ha llegado a ser en una dinámica.

Con la escisión de la disciplina estética del espacio cotidiano, y la homologación de la idea de lo estético al arte, su posibilidad se ha reducido para estetólogos, artistas, o personas de muy refinado gusto, y sus espacios se han delimitado a museos 
la academia. Frente a esto, y como lo expresa Katya Mandoki "no es solo posible, sino indispensable abrir los estudios estéticos -tradicionalmente restringidos a arte y lo bello- hacia la riqueza y complejidad de la vida social en sus diferentes manifestaciones" (Mando,i, 2006: 9). Y es que desde la perspectiv ctiva de esta autora, la teoría estética ha ignorado olímpicamente las prácticas estéticas cotidianas para concentrarse solo en un pomposo juicio que especialistas emiten sobre un minúsculo universo de obras de arte.

"Dado que la estética no es una cuestión exclusivamente filosófica sino cultural, social, comunicativa, politica, económica, histónica, antropotógica, cogritiva, semioica $y$ aun $n$ un trabajo mulidisciplinario puesto a ue varias de esun trabajo multidisciplinario puesto que varias de es
tas disciplinas se traslapan al enfocar estas dinámicas (Mandoki, 2006: 16)

Esta academización de lo estético y reducción de su campo de comprensión a la obra de arte implica

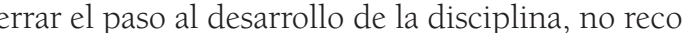
nociendo una de las principales vertientes de este conocimiento: "Al hacer arte, como al hacer ciencia, es imprescindible una actividad lúdica y apareciativa la absorción en el proceso de comprensión con el enorm placer que implica, una admiración por el orden de las formas y las estructuras, un goce en la exploración de incógnitas, la admiración por la elegancia y la simplicidad de soluciones" (Mandoki, 2006: 41), es decir, un contagio de lo cotidiano.

Y es que la estética dice de un sujeto y su relación con el mundo, sensibilidad y abertura a la vida. La valoración de seducción o aversión está regulada en buena medida por convenciones sociales (Mandoki, 2006), esta es una imagen construida, en torno a la cul la dimensión estética como lentes de observación para la intervención social propone nuevas luces para la transformación de contextos golpeados fuertemente por el lado más amargo de la modernidad.

d. El mito de la escisión entre lo subjetivo y lo objetivo en las estéticas tradicionales

Adorno identificará este mito con lo que denomina "postulados tradicionales de la estética". Siguiendo "su propuest ${ }^{4}$, se concibe la estetica pincipalmente desde dos posturas: la primera, como una inter- pretación subjetiva del arte, denominada por él mismo como "estética empírica", filosofía que establece normas estéticas a partir de la clasificación y la generalización de impulsos subjetivos a describir y medir. El empirismo cree que "las obras de arte son haces de estimu en sí mismas está más allá de su capacidad de juicio, incluso de su juicio proyectivo. Solo las reacciones subjetivas ante las obras de arte pueden ser observadas, medidas y universalizadas" (Barahona, 2004: 190). Para Adorno, (1977) "así mismo es como se les escapa lo que forma precisamente el objeto de la estética" (455); la segunda "fenomenología del arte", pretende la existencia originaria de categorías estéticas invariables. Para Adorno, (1977) "la estética no tiene que referirse a su objeto como si se tratara de un fenómeno originario. (...) La fenomenología del arte fracasa por su presupuesto de carecer de presupuestos. El arte se burla de los intentos de elevarse al nivel de pura esencialidad" (Adorno, 1971: 455) La búsqueda de la esencia del arte para Adorno no tiene sentido, pues invisibiliza las constelaciones históricas en las que se constituye. Para Adorno "tanto la estética objetiva como la subjetiva, como polos opuestos, están sometidos a la crítica de la estética dialéctica, la subjetiva porque no es ni trascendentalabstracta ni contingente y dependiente del gusto de cada individuo, y la objetiva porque desconoce la mediación del sujeto en el arte. En la obra, el sujeto no es ni el que la contempla, ni el creador ni el espiritu absoluto, sino el que está atado a la cosa, preformado por ella, sometido a la mediación del objeto" (Barahona, 2004: 219).

La perspectiva estética de Adorno se distancia críticamente de los postulados de la estética tradicional expresada en los anteriores mitos. De cara a sus reducciones, la noción estética propuesta por Adorno se propone como una fuerza crítica de la razón que ha autorizado las peores formas de barbarie, y está más allá del arte como espacio para su expresión: "La deriva estética de la Teoría Crítica, de la que Adorno es el principal guía, responde entonces a la búsqueda de recursos epistemológicos que permitan revisitar la totalidad social y no a un interés secundario de la teoría del arte" (Barahona, 2004: 219).

De esta forma, y a la luz de los mitos presentados, como fuerza crítica, la razón estética nos ofrece:

Como se advierte en el desarrollo argumental de la tesis mencionada me he embarcado en el desafio de abrir la dimensión estéta de la intervención social siguiendo y distanciändome de los planteamientos de la teoría estética en Theodor W. Adorno. Siguiéndolo principalmente en su obra Teoría Estética, publicación póstuma de 1970 - de la cual luego de su muerte en 1969 deja un borrador general- a través de la noción de razón estética, acercamiento marcado por selecciones conceptuales, haciendo una lectura adorniana de lo estético, a partir de elementos encontrados en su teoría respecto del arte. Distanciándose, porque se está muy lejos de que estos sean los planteamientos de adorno. En estos términos, aqui no se pretende reproducir toda la argumentación de la teoria estética de Adorno,
sino, aquellos matices del argumento que se presentan como claves de apertura para la dimensión estética de la intervención social.
1. Una razón crítica que emerge del dolor y lo gestiona fructíferamente. Engancha la apertura de esta dimensión para la intervención social con el propósito último de la teoría estética de Adorno: criticar la barbarie en la cultura en la que ha devenido la humanidad (él es judío y escribe en el marco de la alemania nazi), para posibilitar una sociedad diferente, una sociedad emancipadora. En esta teoría está contenida la promesa de la esperanza de lo otro. Frente a la devastación de la barbarie, lo otro en lo estético -como negaciónimplica una oportunidad de encarnar el deseo de una humanidad liberada: es ante todo un miento ético.

2. La condición dialéctica. La teoría estética de Adorno "se va a caracterizar por mediar el proceso de producción artística con la fuerza conceptual, de tal suerte que no subsuma más lo concreto del trabajo del arte en una serie de conceptos generales y abstractos y en más categorías inmutables" (Barahona, 2004: 198). La estética tradicional niega el carácter dialéctico del arte desde el momento en que en ella no hay una relación efectiva entre pensamiento y objetividad, así como por el hecho de que se manejan en el ámbito de categorías estáticas e inmutables.

3. Una razón emancipadora. Para liberar su verdad, la estética necesita de la cooperación de lo diferente de sí misma: lo mimético, lo que fue sometido y reprimido. Ello se vincula con una razón que debe nutrirse de lo que ella no es, de lo diferente, para vencer la tautología de pensarse siempre solo a si misma.

Como, los alcances de la relectura de la teoría estética de Adorno aquí están más allá del arte, lo que interesa es la fuerza de su racionalidad estética: una razón capaz de abrirse a lo distinto y lo plural logrará romper la lógica de la identidad de lo siempre igual que reprime lo diferente y lo nuevo, y que en definitiva es el estadio de lo mítico, del que la historia quiso liberarse con su proyecto ilustrado para permitir el progreso, y al que ha vuelto a precipitarse derrotada por el totalitarismo (Tafalla, 2003 39 y ss).

De este modo, en Adorno hay una "razón estética" que se propone como alternativa frente a la barbarie en que se ha devenido fruto de la razón dominado-

5 Si bien la noción de dimensión estética es la clave que exploraremos a lo largo de este capítulo, es relevante aclarar que ésta no es una tesis de estética. Lo aqui propuesto se abre desde el Trabajo Social como disciplina para pensar la intervención social. La dimensió estética busca ser un aporte a la construcción de los fenómenos sociales de interés, visibilizando aquello que aün no ha sido visto en la intervención social. ra. Bajo el reconocimiento de la capacidad crítica de la razon estética, pues, "se presta lenguaje a lo que la

\section{Apertura de la dimension estética}

de la intervención social

La dimensión estética en la intervención se planea como un rescate de una razón ampliada para pensar nuestra relación con el mundo, que permit enfrentar la complejidad que las paradojas contemporáneas nos presentan neas de las ciencias sociales: es una lógica a desplegar Siguiendo los planteamientos de Horkheimer, el riesoo de replicar estas formas convencionales de de pensamiento en la intervención social desde está lógica idéntica y no contradictoria, es que no hay cómo volverse "contra el presente cuando el presente es miseria" (Horkheimer, 2000: 139).

Recuperar la aistesis para la intervención de lo social: el camino de la gestión del deseo

Un doctor puede abrir, intervenir, remover entrañas y reubicarlas a voluntad, y el paciente despierta sin más conciencia de ello que los dolores postoperatorios. ¿Qué implica la anestesia? Mediant la anestesia "perdemos el cuerpo", la conciencia, e lugar y sentido en el mundo, es una forma de enajenación (Remedi, 2005).

Una razón anestesiada es la que ha perdido la capacidad crítica, de experimentarse a sí misma, su lugar y sentido, ya no cuestiona el para qué de la razón. Por contraposición, la experiencia estética supon recuperar el cuerpo, recuperar las capacidades facultades humanas (la sensibilidad, la conciencia, la memoria, la razón, la dignidad, la simpatía, la solidaridad, etc.), reencontrarse con el mundo, con sociedad, con nosotros mismos (Remedi, 2005): volver a vibrar con la vida.

Plantear la discusión estética para la intervención social persigue, por lo tanto, enfocar en la cuestión de cómo nos relacionamos con el mundo en el curso del "proceso de la vida" y "nuestro encuentro senen con lo social y con las condiciones materiales espirituales que nos preceden, rodean y constitu(a) agudeza, riqueza, complejidad, etc- - en el mo en que esa forma de estar y relacionarnos con el ideología esconde" (Adorno, 1962: 77).

poustas contemporà- 
mundo nos constituye en tanto sujetos, y específicamente, en tanto actores políticos (Remedi, 2001) Implica abrirse a que el sujeto pueda dibujar su lugar en el mundo, ya no normativizado por imposiciones jerárquicas que le dicen quién tiene que ser. La racionalidad estética como contra-ataque a un razón dominante que autoriza, reproduce y funda modos de vinculación que a diario generan dolo Como plantea (Miranda, 2009) en su investigación. "Transformaciones del referencial normativo de las políticas públicas en sociedades complejas. Observaciones de politicas en Chile" "los procesos de diferenciación funcional generan un descentramiento en los modos de operación de diversos espacios sociales y en sus referenciales normativos. La diferenciación gener autonomía normativa en distintos campos y a distintos niveles, y produce consecuentemente una pluralización de las expectativas normativas de cada espacio. Esto hace que el control social que en la modernidad clásica fue recurrentemente eje sociedad, no logre un ordenamiento e integración de una sociedad crecientemente compleja".

Ello implica la imposibilidad de definir -en el caso particular del proyecto FONDECYT desde la política social- ya sea desde cualquier esfera, a priori un normatividad particularista en la forma de habitar y cohabitar en el mundo.

A pesar del anterior diagnóstico, "las políticas públicas se autocomprenden aún en Chile como el centro rector de la vida en sociedad, mientras que en su entorno una serie de demandas por autonomía operativa y reconocimiento normativo de sistemas funcionales, organizaciones y actores colectivos exigen una visión distinta." (Miranda, 2009). En este sentido, la in( no reconocimiento de esto posiciona a quien inventa la intervención como actor omnisciente capaz de definir a priori todas las consecuencias.

De esta forma, iniciativas que se generan a favor de las personas, se posicionan contra su subjetividad. $Y$ es que "no se puede ni se debe hacer feliz a na contra su voluntad. Porque, en efecto, cuando nosotros decretamos lo que debe ser la felicidad det otro, le estamos arrebatando el valor humano supremo: la libertad de producir su propio orden" (Matus, 2003: 61)

6 De acuerdo con Freud, el inconsciente es la fuente de nuestras motivaciones, las cuales tenemos una tendencia a negar o resistir de su percepción consciente, de manera que solo son observables de forma disfrazada. El Deseo, en el sentido de Freud, el deseo inconsciente, es siempre singular de un sujeto, y no propio de la especie, es un deseo que, a diferencia de la necesidad, no camina en el sentido de la supervivencia y la adaptación. Es un deseo que por el contrario daña, es al mismo tiempo un deseo indestructible, un deseo que no se
puede olvidar porque es esencialmente insatisfecho. A diferencia de la necesidad, no es una función vital que pueda satisfacerse, pues en puede olvidar porque es esencialmente insatisfecho. A diferencia de la necesidad, no
su surgimiento mismo esta coordinado con la función de la pérdida. (CASTRILO)
La intervención no está en condiciones de recomendar ni imponer modelos de vida lograda a seguir, ello es parte del camino de la emancipación.

\section{Gestionar el deseo en la intervención social}

Hablar de deseo en el mundo moderno parece ser problemático, y ello repercute también en la intervención social. Desde el freudismo ${ }^{6}$-bajo la apariencia de una ciencia- se construyó un mito, a saber: el mito de una necesaria castración del deseo, su sumisión al triángulo edípico, una interpretación significante que tiende a separar el análisis de sus implicaciones sociales reales (Guattari, 1989).

El deseo otras veces es también reducido a un tema sexual, o la expresión de aquel espacio indómito, irracional, de las pulsiones de vida y de muerte. Así, en una sociedad que se esfuerza por moverse en un marco "racional", el deseo es amputado, banalizado y clausurado como camino. La sociedad moderna -en tanto traiciona al proyecto ilustrado- cierra los no un lugar donde se juega la posibilidad de lo humano. deseo. Los medios de comunicación se convierten entonces en el zócalo del sistema y de cierto monopolio económico, pasando de ser dibujo de lo social a dibujantes de lo social.

Así, nos volvemos -en términos de Guattari- en máquinas deseantes, serviles al sistema. Con la Ilustración se pretende salir de la servidumbre a la Iglesia, a los tutelajes impuestos que nos dejan cono "menores de edad". Pero en vez de ello, y a volvemos ahora siervos de un modo de vida totalizado en valoraciones de la razón instrumental. Esta nueva servidumbre (Guattari, 1989) es una servidumbre mecánica -anestesia-. El ideal (de la sociedad actual) ya no es tener que ver con individuos ricos en pasiones, capaces de ambigüedad, 1989). Desde esta perspectiva, cuando un niño pide espacios para la exploración del deseo. El deseo se ha vuelto también una herramienta de dominio, ya

De este modo, se construyen dibujos mediáticos del punto de proliferación y de creación posible, en el seno de un sistema constituido (Guattari, 1989).

Razón dialectico-estética y gestión del deseo en

Bajo la mirada crítica emerge la posibilidad de hacer aparecer otras nociones de deseo, para romper con ello el mito que ha reducido -casi a la inexistencia- la posibilidad de lo humano. Y es que si el humano se asume como un proyecto inacabado, es en la posibilida de autodeterminarse que se ga su humanidad; ¿Cuáles son las posibilidades de autodeterminación como sujeto que ofrece la intervención social? Esta posibilidad no está disponible a primera mano, es en estos términos una gestión, un trabajo a desarrollar: en tanto ha sido petrificado y naturalizado, su movimiento para una construcción emancipatoria del sujeto es un trabajo a sostener.

En este sentido, el deseo es también racional, pero para así concebirlo "se hace preciso una noción de sujeto que no lo deje ni inocente ni inactivo frente a su propia pasión. Que no lo exima de su exploración que no es otra cosa, que el sí mismo desplegándose "(Matus (b)". Lo humano es entonces vivido como un combate, contienda que puede asumir la actitud y la tarea del ideario ilustrado, del desafío humano contenido en ella. "(Matus (b), s/f)".

\section{Algunas implicancias para la gestión}

\section{del deseo en la intervención social}

Presento finalmente algunos elementos iniciales para posibilitar la gestión del deseo en la intervención social. Resalto la condición de iniciales, pues el trabajo de esta dimensión recién comienza.

\section{Romper las naturalizaciones}

Como ya hemos adelantado, muchas veces los lugares sociales en que le toca desenvolver su vida a los sujetos que se acoplan a los procesos de intervención social ofertados están naturalizados. ¿Qué implica esto? Que pensamos que los fenómenos sociales responden a una esencialidad donde -por ejemplo- se "es" pobre, se habla de "la" pobreza, y se le asignan por identidad las valoraciones sociohistóricas de una categoría a una persona natural. Abrir la gestión del deseo desde la dimensión estética en la intervención social requiere de un primer movimiento desnaturalizador: y es que la mayor dominación está en el olvido.

Hay al menos 3 ámbitos donde se juega esta posibilidad en tanto desnaturalización: a. Respecto del fenómeno de intervención; b. Respecto del sujeto de intervención; c. Respecto del modo de intervención. Se colocan algunos antecedentes de ellos.

\section{a. Respecto del fenómeno}

Implica reconocer la identidad en la no-identidad entre fenómeno y concepto. La identidad, en tanto el fenómeno es histórica-conceptualmente construido, y la no identidad, en tanto el fenómeno no se reduce al concepto, contiene en sí la posibilidad de lo otro, posibilidad que es también conceptual.

El extremo negativo de esta clave se encuentra en el mito de la ontologización, donde se homologa el concepto al fenómeno, y se genera una inmovilización de la categoría, adquiriendo esta relación un condición de segunda naturaleza. La naturalización del orden social dado parece ser una verdad revelada incuestionable, al estilo de aquellas verdades míticas que no encontraban explicación pues simplemente "así era".

En este sentido, al no reconocer que lo que hay detrás de cualquier sociedad es una forma de ver y capturar el mundo, se imposibilita la existencia sistema de comprenson sola consituido at menos por cuatro dimensiones ${ }^{7}$ constituido al menos por cuatro dimensiones ${ }^{7}$ relacionadas aunque no homologables, siendo una de ellas, y quizás una de las más importantes, el marco ético/valórico en que se genera la propuesta.

De este modo, el esfuerzo se dirige a encontrar ojos para ver, palabras para conformar un lenguaje, heramientas para deconstruir discursos, vías para adentrarse en las contradicciones de eso que denominamos realidad social, develando su régimen de la mirada (Foucault, 1996). Sin la exploración de esta mirada, la intervención social en Trabajo Social no es más que la reproducción de lo existente.

\section{b. Respecto del sujeto: Romper la}

\section{naturalizaciones en la forma de pensar al otro}

Implica reconocer la identidad en la no identidad entre sujeto y concepto. No se puede homologar ni reducir al sujeto al concepto generado sobre este, ni se puede homologar a la persona natural a la categoría construida como sujeto en la intervención. La mímesis conceptual para el sujeto implicará por tanto romper estas dos reificaciones: la del concept sobre el sujeto y la del sujeto conceptual sobre la persona natural De esta forma, el concepto aparece como contingente, y la persona como no idéntica a 
la categoría conceptual. El sujeto es un proyecto a construir, y la gestión del deseo es una clave de su despliegue. Esta ruptura asume al sujeto como un proyecto inacabado.

\section{c. Respecto del modo de intervención: Romper las} naturalizaciones en la forma de trabajar con el otro Implica reconocer el carácter mítico en que esta ha devenido, para criticarlo: trente a las reduccionesen 8 , se vise las estrate elevante la concordancia entre la constelación explicativa construida y la estrategia de intervención desplegada. En este sentido, se vuelve clave apostar por la creatividad a la hora de pensar los mecanismos de intervención: abrirse a la diversidad en los modos de hacer; incorporar la interdisciplinariedad como una fuerza productora: visibilizar la riqueza de la vida cotidiana y dejar que se exprese en formas de trabajo desplegadas.

Frente a los tres espacios expuestos, la desnaturalización en el concepto se presenta como e reconocimiento de la intervención social en tanto comunicación contingente, donde el lenguaje juega un papel clave Desde lo anterior, lo estético en el apuesta para romper con las naturalizaciones que se generan al nombrar lo social en la intervención social, que llevan a estigmatizar aquellos fenómenos con los que se interviene, tant en sus conceptualizaciones del fenómeno, como de sujeto y sus estrategias desplegadas. El esfuerzo está puesto en generar un discurso que genere condiciones de simetría.

Detrás de estas tres dimensiones está de fondo lo que la teoría crítica denominará "el trabajo del concepto". El concepto es visto aquí como un resultado, no como el punto de partida. Implica recorre un camino de determinaciones múltiples. El conocimiento, por tanto, implica saber diferenciar.

En este sentido, frente a la reducción identitaria-no contradictoria del pensamiento reinante, emerge el camino del trabajo del concepto en un horizonte de construcción de constelaciones: "Lo urgente para el concepto es aquello a lo que no llega, lo que el mecanismo de su abstracción elimina, lo que no es de antemano un caso de concepto" (Adorno, 1975: 16).

Es en este sentido, que el trabajo del concepto se vuelve relevante pues "La mediación conceptual se ve desde su interior como la esfera más importante. sin la que es imposible conocer; pero esa apariencia no debe ser confundida con su verdad" (Adorno, 1975

20). El trabajo del concepto entonces permitirá revelar este orden natural mitificado. Solamente a través de la agrupación de conceptos en torno al objeto a interpretar, se puede devenir en conceptos concretos.

Romper la naturalización de los conceptos. Volver a dar movimiento, cuando las cosas parecían ya conocidas, hacerlas aparecer como nuevas y enigmáticas. Ese es el desafío de la comprensión de lo social y su intervención: La constelación como mecanismo de apertura de los fenómenos sociales permitirá descifrar una realidad contradictoria.

2. Ampliar el espectro de las posibilidades posibles Desde la teoría funcionalista, no todas las posibilidades disponibles en el entorno son posibles de actualizar por un sistema. Los mitos de que todo es posible se han instalado en la intervención social, decantan en modos de hacer que delegan las resy decantan en modos de hacer que delegan las responsabilidades de "no participación" en una oferta disponible en los sujetos afectados: el que quiere puede llegar a la universidad, superar la pobreza salir del círculo de la violencia doméstica, etc.

De aquí la idea de pensar la intervención como un dispositivo de apertura de las posibilidades posibles de actualizar por el sistema.

El desafío de la gestión del deseo entonces, está en ampliar el arco de posibilidades, aumentar la libertad de los sujetos, de modo de que estas posibilidades sean posibles de actualizar. La intervención social -como espacio de transformación del contexto- puede incorporar en mayor o menor medida las aspiraciones y sueños de sus usuarios. Ellos constituyen expresión del deseo.

Como hemos intentado argumentar con la crítica a la participación y el posterior argumento desplegado, el deseo no es accesible a primera mano, el deseo se trabaja en la intervención, pues ha sido mediatizado.

El trabajo de la exploración del deseo se constituye entonces en la apertura del arco de posibilidades de los sujetos. Por ejemplo, para el caso de las rejas de Proyecto Propio donde ellos solo veían rejas, poder visualizar otras alternativas. Nadie deseará algo que no conoce, algo que no está en sus posibilidades. Explorar el deseo, abrir espacios para él en esta intervención es abrir este arco de posibilidad. La intervención, entonces, desplegará mecanismos para que el sujeto explore su deseo. De esta forma, para desplegar el potencial estético se requiere de una intervención que movilice y recoja los sueños, las aspiraciones y los proyectos y los plasme como propuestas posibles (Matus, (b), s/f)

\section{El sujeto como proyecto inacabado}

Frente a la búsqueda de la integración a través de la uniformación de los sujetos, la gestión del deseo se ofrece como ejercicio crítico contra el orden bárbaro que se ha establecido. Desde la perspectiva traría "fundamentado en un imaginario ideológico (tecnológico) que subordina la práctica profesional a visiones externalistas de manipulación, control o cosificación de las personas y situaciones" (Vélez, 2003: 54). No hay posibilidades para la autodeterminación individual -y menos- colectiva. Requiere disponer de la posibilidad de incorporar sus propios proyectos de vida lograda el suje el sujeto pueda decidir su propia vida. Una intervención predefinida en procesos y contenidos por el experto, va a imposibilitar a los usuarios el derecho ético que cada uno tiene a decir su propia vida. Como indica Rosa Cañete, (2006) a propósito del tema de la equidad -además de por la igualdadequidad pasa por el derecho a la diferencia: "Garantizar que no haya una sola opción donde deben existir diferentes opciones, de tal forma que operen respuestas sociales para que la igualdad sea ajustada a la diversidad de realidades y aspiraciones de cada sujeto" (Cañete, 2006).

Como plantea Habermas, hay que concederle a los sujetos un cierto privilegio en lo que atañe a la interpretación de sus propias necesidades (Mc Carthy, 1992: 200 y ss). Una intervención que busca desplegar su potencial estético no puede ser prescrita, inflexible e impersonal. No abrir este espacio, implica reproducir la lógica de la minoría de edad, donde los sujetos son puestos como vasallos incapaces de autodeterminarse como comunidades.

En términos de las intervenciones sociales, la negación del espacio de la autodeterminación -individual y colectiva- se vuelve particularmente perjudicial: el político y el experto parecen ser los únicos que saben lo que le conviene al pueblo, los ciudadanos son incompetentes básicos en la toma de decisiones" esto exige una aceptación y sumisión ciega, esto es irracional. Como indica Adela Cortina: (1999) "Los pobres son hoy aquellos interlocutores virtuales, que nunca serán interlocutores reales" (93).

El desafío está puesto en "no seguir enfatizando mecanismos que perpetúan y socavan las posibilidades autorregulatorias de los propios sujetos" (Matus, 2003: 64). Se relaciona con la búsqueda de un proyecto de democracia. "en el que cada individuo o grupo pueda perseguir su concepción del bien, permitan un proceso público de legitimación, deslegitimación y cambio de las interpretaciones y regulaciones que las especifican y aseguren una textura social en la que la posibilidad de perseguir la propia concepción del bien sea efectiva" (Habermas, 1991: 53)

4. Que se abra a lo inesperado, rompiendo la ilusión del control

Implica romper el delirio de guiar por el buen camino a "personas" incompetentes para resolver su vida: por algo son pobres. Implica abrirse a la posibilidad de que lo inesperado pase, ofreciendo desde la procedimentalización un marco de acción que contenga y recupere lo mejor de la fuerza creativa de su vida cotidiana.

La noción de procedimentalización abre posibilidades en estos términos: el diagnóstico que se hace es que las intervenciones convencionales están "llenas de sustancia". En términos habermasianos, esto refiere a que se encuentran cargadas de contenido normativizante, que reducen las posibilidades de la diferencia como espacio legítimo de expresión.

En este sentido, emerge el desafío para los profesionales que intervienen lo social de caminar hacia un indicación procedimental de la intervención, vacío de este contenido, entendiendo esto no como una totalización impositiva, sino como apertura de posibilidad. No conlleva respuestas materiales dadas a priori.

a procedimentalización implica "superar tanto perspectivas inductivas como deductivas, así como no precisar de una definición apriorística de contenidos, ya que ellos no se fijan por fuera de una interacción con los propios sujetos. En este sentido, no conlleva respuesta material dada como un a priori, ya que esta han de buscarla los agentes morales mismos y nadie puede buscarla por ellos. Con esto, lo moral se inscribe en las estructuras de la interacción, en la medida en que permite que la razón no se considere como un principio originario externo, una suerte de orden objetivo sino que se ancle en la misma estructura de la práctica argumenlativa" (Matus (a) s/f), 75).

Procedimentalizar es equivalente a dibujar la cancha para que el otro juegue, desplegándose.

\section{La condición utópica}

Como ya denunció Nietzsche, en un mundo donde reina la alienación y la cosificación es necesario un arte perturbador, reacio a convertirse en mera distracción; esto ya que más que a otras maniestaciones del espíritu humano, se le atribuye 
precisamente al arte la capacidad de hacer justicia a lo inefable, a lo inexpresable

De esta forma el pensamiento no-identitario y contradictorio postulado por la teoría crítica, genera un espacio para la dimensión utópica. Esto en el sentido de que existe una dimensión utópica de la no identidad referida a lo concreto "la transitoriedad de los particulares era promesa de un futuro diferente, mientras que su pequeña dimensión, su resistencia a la categorización implicaba un desafio a la misma estructura social que expresaban" (Buck Morss, 1981: 167). Esta dimensión de lo utópico no es viable en un marco de pensamiento tradicional, donde lo utópico es visto como "una pérdida de tiempo, pues no es viable". En un marco de orden social naturalizado, hay que pensar dentro de los límites de lo pensado por lo que bajo esta restricción lo utópico carece totalmente de sentido.

Ahora bien, en el pensamiento crítico, y bajo las posibilidades que abren sus principios de no-identidad y contradicción, la posibilidad de pensar lo no pensado juega un rol clave como reconocimient de la transitoriedad de lo actual, que si es de un modo, contiene las mismas posibilidades de ser de otro distinto, las cuales tienen que ser exploradas, evidenciadas, desmitificadas.

La posibilidad abierta desde el pensamiento crítico para lo utópico es clave para la generación de una intervención social transformadora y que genere progreso crítico. Si el pensamiento idéntico ha clausurado el camino de lo otro, lo utópico, la intervención social pierde sentido. La posibilidad de la utopía que abre el pensamiento dialéctico estético en la interv nos colectivos. Una intervención social sin utopía no se vuelve más que una mala representación vacía, representante del sistema.

\section{Gestionar el dolor}

La teoría estética de Adorno emerge en el dolor. Ella no representa una simple faceta temática dentro de un discurso filosófico general, sino el último recurso de una filosofía que enmudece ante el desastre (Castillo, 2003). Este asunto no es menor: es un punto de encuentro con los fenómenos sociales que queremos enfrentar a la categoría de gestión del deseo desde la intervención social. Las comunidades que habitan bajo las categorías que se levantan sobre estos fenómenos sociales muchas veces asumen un fuerte carga de dolor y frustración: mujeres golpeadas; familias pobres; niños vulnerados; trabajadores insatisfechos; ancianos abandonados; etc.
Nuevamente la posibilidad interpretativa que nos ofrecen las propuestas contemporáneas en ciencias sociales abren aquí posibilidades de relectura fructíferas. La gestión del deseo debe ser capaz de trabajar el dolor, y ponerlo al servicio de su potencial movilizador. Como la teoría crítica nos ha adelantado: el poder del desencanto fructífero.

\section{Fuentes}

ADORNO, T. (1975). "Dialéctica negativa", Madrid, Taurus.

ADORNO, T. (1962). Noten zur Literatur I, Barcelona, Ariel.

ADORNO, T.W. (1971). "Teoría estética" Ed. por Gretel Adorno y Rolf Tiedmann; trad por F. Riaza. Madrid Taurus.

BARAHONA, A. (2004). "Teoría de la racionalidad y crítica social en Theodor W. Adorno: Utopía y razón dialécticoestética en su filosofía." Memoria para optar al grado de doctor. Universidad Complutense, Facultad de Filosofía. Madrid.

BUCK, M. (1981). Origen de la dialéctica negativa: Theodor W. Adorno, Walter Benjamin y el Instituto de The Siglo Veintiuno.

CAÑETE, R. (2006). "Notas de clase" IV Diplomado en Desarrollo Humano, CEPAL 26 octubre.

CASTILLO, G. (2003) Negatividad, esperanza y margen en la estética de Theodor W. Adorno. Revista Aisthesis

CORTINA, A. (1999). "Los ciudadanos como protagonistas" Barcelona Galaxia Gutenberg.

FOUCAULT, M. (1966). "El nacimiento de la clínica". Editorial Siglo XXI. México.

GUATTARI, F. (1989). "Cartografias del deseo"; traducción de Miguel Denis Norambuena. Santiago, Chile Francisco Zegers.

HABERMAS, J. (1991). Escritos sobre moralidad y eticidad. Paidó

HORKHEIMER, M. (2000). "Teoría tradicional y Teoría crítica" Editorial Paidos Ibérica.

KANT. (1960). "Crítica de la razón pura" Buenos Aires: Losada.

MC CARTHY, T. (1992). Ideales e Ilusiones. Reconstrucción y deconstrucción en la teoría crítica contemporanea. Tecnos, Madrid.

MANDOKI, KATIA. (2008): "Estética cotidiana y juegos de la cultura". Editorial Siglo XXI.

MATUS, T. (a) (s/f) "Apuntes sobre intervención social". Documentos sin publicar.
MATUS, T. (b) (s/f) "Sujeto y pasión". Las posibilidades estéticas de una exploración tensional como elementos para el combate por lo humano. Documentos sin publicar.

MATUS, T. (2004). "La reinvención de la memoria : indagación sobre el proceso de profesionalización del trabajo social chileno 1925-1965" Pontificia Universidad Católica de Chile, Facultad de Ciencias Sociales, Escuela de Trabajo Social, Santiago, Chile.

MATUS, T. (2003). "La intervención social como gramática. Hacia una semántica propositiva del Trabajo Social frente

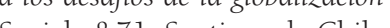

MATUS, T. "Sujeto y pasión: Las posibilidades estéticas de una exploracion tensional como elementos para el combate por lo humano".

MATUS, T. (1999). "Propuestas contemporáneas en Trabajo Social: hacia una intervención polifónica" Editorial Espacio- Buenos Aires.

MATUS, T. (1994). "Cuando la Epistemología no basta" Revista Acto Social n” 4, Córdoba, Argentina.

MIRANDA, P. (2009). Proyecto FONDECYT No 1095186 "Transformaciones del referencial normativo de las Politicas Públicas en sociedades complejas. Observaciones de politicas en Chile".

CASTRILO D. "Diccionario crítico" Universidad Complutense de Madrid disponible en: http://www.ucm.es/

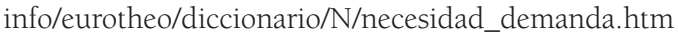

REMEDI, G. (2005). "Las bases estéticas de la ciudadanía" en Revista Aisthesis $n^{\circ}$ 38. Instituto de Estética Pontificia Universidad Católica de Chile. Año.

SÁNCHEZ, J. (2007). "Los dibujos mediáticos del deseo" Espéculo. Revista de estudios literarios. Universidad Complutense de Madrid. 2005 Disponible en http:// www.ucm.es/info/especulo/numero30/dideseo.htm

SERRANO, C. (1998). "Participacion Social y Ciudadanía: Un debate del Chile contemporáneo" Asesorías para el desarrollo.

TAFALlA, M. (2003). "Theodor W. Adorno, Una filosofia de la memoria". Barcelona: Herder

VÉLEZ, O.L. (2003). Reconfigurando el Trabajo Social. Perspectivas y tendencias contemporáneas. Editorial Espacio Buenos Aires. Argentina. 